\title{
Intervenciones Humanitarias para la Recuperación del Trauma con Terapia EMDR en Latinoamérica y el Caribe
}

\author{
Ignacio Jarero \\ Lucina Artigas \\ Susana Uribe \\ Alaide Miranda \\ Fundación Latinoamericana y del Caribe para la Investigación del Trauma Psicológico. \\ Latin American \& Caribbean Foundation for Psychological Trauma Research.
}

\begin{abstract}
Este artículo presenta un resumen de las Intervenciones Humanitarias para la Recuperación del Trauma con Terapia de Reprocesamiento y Desensibilización a través del Movimiento Ocular (EMDR) en Latinoamérica y el Caribe y proporciona al lector ejemplos de historias clínicas obtenidas en la primera línea de apoyo. A lo largo de los muchos años realizando trabajo de campo, hemos observado que el trauma psicológico, como consecuencia de las situaciones multifacéticas que enfrentan los individuos y comunidades después de un desastre, implica un gran reto. En el presente artículo, describimos las Intervenciones Humanitarias con Terapia EMDR realizadas desde 1998 en Latinoamérica y el Caribe, para tratar las perturbaciones psicológicas que se presentan en las y los sobrevivientes después de desastres naturales (ej. inundaciones, deslizamientos de tierra, terremotos), desastres provocados por el hombre, masacres humanas y violencia interpersonal severa. Se ha proporcionado tratamiento a niños, adolescentes y adultos sobrevivientes, frecuentemente en las comunidades donde ocurrieron los desastres; así como a auxiliadores y a pacientes con cáncer. Los protocolos de Intervención Temprana con Terapia EMDR son intervenciones breves y efectivas que pueden ser utilizadas en campo o en situaciones de emergencia. Existe un cuerpo de investigaciones que apoyan el uso de protocolos modificados de Terapia EMDR para tratar el trauma agudo en formatos de atención individual y grupal (Jarero, Artigas, \& Luber, 2011).
\end{abstract}

Palabras Clave: Terapia de Reprocesamiento y Desensibilización a través del Movimiento Ocular (EMDR); Protocolo Grupal e Integrativo con Terapia EMDR (EMDR-IGTP); programas humanitarios para la recuperación del trauma, masacre humana; pacientes con cáncer; violencia interpersonal severa.

atinoamérica ha sufrido desastres naturales como inundaciones, huracanes, terremotos catastróficos y erupciones volcánicas. Además, desde los años setenta, ha sido víctima de altos niveles de violencia política, la cual aumentó a un punto máximo hasta tomar forma de guerras, insurgencias, contrainsurgencias y otros conflictos civiles en los años ochenta. Las consecuencias de esto fueron: violencia extrema, masacres humanas, continuas desapariciones, violaciones, torturas y quema de pueblos (Norris, Murphy, Baker \& Perilla, 2004).
De acuerdo con Biles y Cobos (2004), Latino américa es una de las zonas de más alto riesgo en el mundo debido a que geográficamente está situada sobre una de las principales placas tectónicas con actividad sísmica regular. Del 2006 al 2010, una cuarta parte de todos los desastres naturales -442 de 1915- sucedieron en Latinoamérica. El número de habitantes afectados fue de 48 millones, los cuales son un 5\% de los 904 millones de personas afectadas a nivel global (Organización Panamericana de la Salud, 2012).

This is a modified translation of an article originally published as Jarero, I., Artigas, L., Uribe, S., \& Miranda, A. (2014). EMDR Therapy Humanitarian Trauma Recovery Interventions in Latin America and the Caribbean. Journal of EMDR Practice and Research, 8(4), 260-268. Translated by Ignacio Jarero. 
En una encuesta realizada recientemente a 858 estudiantes de cuatro universidades públicas de México (de edades entre los 18 y 25 años), situadas en una de las muchas ciudades que son afectadas por la guerra entre carteles de drogas, que pelean por el control de las zonas en las que se producen drogas sintéticas; los investigadores encontraron una prevalencia del $78 \%$ de eventos traumáticos considerados como impactantes para la integridad física y emocional de los encuestados. El evento con mayor prevalencia fue la muerte repentina de un miembro de la familia o un amigo cercano (Mojica, Márquez, Guadarrama \& Ramos, 2013)

\section{La Terapia EMDR}

La Terapia de Reprocesamiento y Desensibilización a través del Movimiento Ocular (EMDR) fue desarrollada por la Dra. Francine Shapiro (2001) y es una aproximación terapéutica integral para el tratamiento del trauma, experiencias adversas de vida o estresores psicológicos, que consta de ocho fases y tiene como guía al modelo del Sistema de Procesamiento de la Información a Estados Adaptativos (SPIA).

El marco teórico del SPIA, en el que está basado esta psicoterapia, ilustra las amplias posibilidades de aplicación del tratamiento. En la Terapia EMDR, las memorias almacenadas disfuncionalmente, son consideradas como la base primaria de la patología clínica no causada por un déficit orgánico. Se postula, que el reprocesamiento de estas memorias, conduce a su resolución a través de su reconsolidación y asimilación dentro de redes de memoria adaptativas más extensas (Oren \& Solomon, 2012).

La Terapia EMDR es recomendada para el tratamiento del Trastorno por Estrés Postraumático (TEPT) en niños, adolescentes y adultos por la Organización Mundial de la Salud (2013) y por numerosas directrices internacionales, como la Cochrane Review (Bisson \& Andrew, 2007). También se dice que es aplicable a un amplio rango de padecimientos clínicos basados en experiencias perturbadoras (Shapiro, 2001; Solomon \& Shapiro, 2008).

\section{Intervención Temprana con Terapia EMDR}

La experiencia clínica y el trabajo de campo administrando Intervención Temprana con Terapia EMDR (EEI, por sus siglas en inglés) es extenso (Maxfield, 2008). Los resultados de estudios preliminares indican que la EEI es una intervención breve, con efectos de tratamiento rápidos, que puede ser utilizada en campo o en situaciones de emergencia (ej. Jarero, et al., 2011).
Iniciativas de Investigación

Se requieren estudios aleatorios y controlados para investigar y evaluar el tratamiento de incidentes críticos. Esto permitiría desarrollar y aplicar terapias efectivas para aliviar el sufrimiento de las muchas víctimas de desastres que existen en el mundo (Luber, 2009). El tratamiento de las perturbaciones psicológicas que se presentan a partir de un evento traumático es un reto para Latinoamérica y el Caribe, pues existe una falta de recursos profesionales que puedan responder adecuadamente a eventos traumáticos de gran magnitud y existen pocos psicoterapeutas con entrenamiento específico en el tratamiento del estrés postraumático.

Además, la gran movilidad de los sobrevivientes de desastres, hace muy complejo realizar investigaciones en las que se puedan observar los resultados del tratamiento. Sin embargo, para nosotros se ha convertido en una prioridad el investigar la efectividad de los protocolos de Intervención Temprana con Terapia EMDR, en todo momento en que las circunstancias sean apropiadas para ello. Nosotros creemos que la investigación puede alimentar de información y dirigir las intervenciones, permitiéndonos mejorar y crear estrategias adecuadas de tratamiento en estas difíciles situaciones.

El Protocolo Grupal e Integrativo con Terapia EMDR. El Protocolo Grupal e Integrativo con Terapia EMDR para intervención temprana (EMDR-IGTP, por sus siglas en inglés; Artigas, Jarero, Alcalá \& López Cano, 2014), fue desarrollado por miembros de la Asociación Mexicana para Ayuda Mental en Crisis (AMAMECRISIS). Éste combina el protocolo estándar de ocho fases de la Terapia EMDR (Shapiro, 2001), con un formato de terapia grupal y arte-terapia y utiliza el abrazo de la mariposa (Artigas \& Jarero, 2014) como una manera de auto-administrarse estimulación bilateral.

Es una intervención altamente eficaz en términos de tiempo, costos, recursos y resultados a largo plazo. Este protocolo se ha utilizado en su formato original o con modificaciones para adaptarse a las circunstancias culturales en numerosos sitios alrededor del mundo (Gelbach \& Davis, 2007; Maxfield, 2008) y para tratar a miles de sobrevivientes de desastres naturales o provocados por el hombre (Jarero \& Artigas, 2009, 2012).

La evidencia científica que comprueba su eficacia se ha obtenido de nueve estudios piloto que fueron llevados a cabo en proyectos humanitarios con niños y/o adultos después de desastres naturales ocurridos en México, Nicaragua, El Salvador, Colombia y Venezuela (Artigas, Jarero, Mauer, López Cano \& Alcalá, 2000; Jarero, Artigas \& Hartung, 2006; Jarero, Artigas, Mauer, López Cano \& Alcalá, 1999). Además, reportes de caso y estudios de campo realizados 
alrededor del mundo, han documentado su efectividad con niños y adultos después de desastres naturales o provocados por el hombre, durante traumas de guerra en curso, crisis geopolíticas, con niños refugiados de guerra, en accidentes de trabajo que han producido trastorno por estrés agudo, con víctimas de violación y con niños y niñas víctimas de violencia interpersonal severa (ej. Fernández, Gallinari, \& Lorenzetti, 2005; Jarero \& Artigas, 2010; Zaghrout-Hodali, Alissa, $\&$ Dodgson, 2008).

Otros Protocolos de Intervención Temprana con Terapia EMDR. El Protocolo de EMDR para Incidentes Críticos Recientes (EMDR-PRECI, por sus siglas en inglés), es un protocolo modificado de Terapia EMDR en su modalidad individual. Se desarrolló en campo para tratar incidentes críticos (ej. terremotos, inundaciones, deslizamientos de tierra) relacionados con eventos estresantes que continuaron por un largo período de tiempo y en los que no hubo un período de seguridad post-trauma para la consolidación de la memoria. Existe evidencia preliminar que apoya la eficacia del EMDR-PRECI en la reducción de síntomas de estrés postraumático en adultos, manteniendo sus efectos a pesar de amenazas y peligro constante; dentro del contexto del continuo de cuidados en salud mental en desastres y como intervención temprana para personal forense traumatizado (Jarero et al., 2011; Jarero \& Uribe, 2011, 2012).

El Protocolo Individual de Terapia EMDR para uso de Paraprofesionales en Situaciones de Trauma Agudo (EMDR-PROPARA, por sus siglas en inglés), es una adaptación del EMDR-PRECI para uso de paraprofesionales. Existe evidencia preliminar que sustenta su efectividad en la reducción de la severidad de los síntomas de estrés postraumático y en el mejoramiento global subjetivo (Jarero, Amaya, Givaudán \& Miranda, 2013).

\section{Intervenciones Humanitarias con Terapia EMDR en Latinoamérica y el Caribe}

Las intervenciones humanitarias para la recuperación del trauma con Terapia EMDR iniciaron en Latinoamérica en el año 1998, con el objetivo de enfrentar la gran necesidad de servicios de salud mental, que se requirieron después de que el Huracán Paulina devastó las costas de los estados de Oaxaca y Guerrero en México en el año 1997.

El Equipo humanitario de Terapia EMDR consistió en 17 terapeutas mexicanos y 3 terapeutas extranjeros: 1 canadiense y dos estadounidenses (todos miembros de EMDR-HAP-USA) que fueron invitados a colaborar con el equipo mexicano. Cuando llegaron al sitio del desastre se encontraron abrumados por el número de sobrevivientes que requerían apoyo. El EMDR-IGTP y el abrazo de la mariposa fueron creados durante este proyecto, porque era imposible aplicar el Protocolo de Terapia EMDR en su modalidad individual, a la gran cantidad de personas que lo necesitaban, con el poco tiempo del que se disponía. Durante 3 semanas el equipo trabajó in situ y trataron a un total de 273 sobrevivientes.

Organizaciones que Proporcionan Ayuda

Humanitaria con Terapia EMDR en

Latinoamérica y el Caribe

AMAMECRISIS. La Asociación Mexicana para Ayuda Mental en Crisis (AMAMECRISIS), fue legalmente constituida bajo las leyes mexicanas como una Organización No Gubernamental (ONG) en 1998. El mandato de los programas de esta ONG, es llevar a cabo intervenciones humanitarias para la recuperación del trauma (IHRT) en poblaciones de bajos recursos. Todo el personal trabaja como voluntario y la mayor parte del financiamiento proviene de los entrenadores mexicanos de terapia EMDR, quienes donan el 50\% de las ganancias de los entrenamientos que imparten.

A través de los años, AMAMECRISIS ha realizado IHRT a lo largo de la República Mexicana, Colombia, Haití, Nicaragua, El Salvador, Venezuela y España; y ha ofrecido asistencia a terapeutas EMDR de otros países alrededor del mundo, para ayudar a aquellos que han sobrevivido algún desastre.

Hace 5 años AMAMECRISIS se afilió a la Fundación Latinoamericana y del Caribe para la Investigación del Trauma Psicológico (Ganadora del Premio Francine Shapiro a la Investigación) para fortalecer el programa humanitario de recuperación del trauma y realizar investigación de una manera más eficaz.

Otras ONG Latinoamericanas. Las Comunidades de Terapia EMDR en Argentina y Brasil han constituido ONGs para proporcionar ayuda humanitaria con Terapia EMDR. La Asociación EMDR-HAP Argentina (Adúriz, Knopfler, Bluthgen, 2009; Aduríz \& Salas, 2014), el Centro de Psicoterapias de Argentina (Salas, 2014) y EMDR Iberoamérica-HAP-Brasil (Monteiro, 2014), han llevado a cabo y publicado sus intervenciones humanitarias después de desastres naturales en sus países.

Tipos de Intervenciones para la Recuperación del Trauma Ilevadas a cabo por AMAMECRISIS

Tratamiento. Los miembros del equipo humanitario para la recuperación del Trauma de AMAMECRISIS son voluntarios y siempre proporcionan el 
tratamiento de manera gratuita en el lugar del desastre, de acuerdo a las fases de recuperación y a la evaluación de las necesidades que los sobrevivientes estén experimentando. El equipo llega al lugar del desastre cuando las circunstancias lo permiten (ej. seguridad, alimento, estancia). Algunas veces esto puede ocurrir 15 días después del incidente crítico o algunas veces después de 3 o 4 meses.

AMAMECRISIS tiene un sistema integral de intervenciones post-desastre que sigue un continuo de cuidados - una progresión de cuidados de la salud que se van intensificando paulatinamente. Este modelo de intervención terapéutica está basado en dos grandes abordajes: el manejo de estrés en incidentes críticos (modelo de la International Critical Incident Stress Foundation, CISM) y los Protocolos de Terapia EMDR.

Los servicios son proporcionados por terapeutas expertos y terapeutas en capacitación bajo su supervisión. Es obligatorio que todos los miembros del equipo sigan los lineamientos de auto-cuidados de la Green Cross Academy of Traumatology (2008) debido a que "las conmovedoras historias de los sobrevivientes, lo que se ve, los olores, los sonidos y las emociones que asaltan los sentidos del personal de salud mental, pueden ir desde lo incómodo hasta lo abrumador" (Jarero \& Uribe, 2014, p. 533) y pueden afectar severamente sus vidas.

Entrenamiento de los Terapeutas Locales. Después de llevar a cabo las intervenciones humanitarias tempranas con Terapia EMDR, los entrenadores del Instituto EMDR y EMDR-Iberoamérica, ofrecen entrenamientos gratuitos (básicos y avanzados) dirigidos a profesionales de la salud mental locales, con el objetivo de que éstos puedan ofrecer Terapia EMDR a largo plazo en su comunidad. Los entrenadores también les brindan apoyo para que formen su propia Organización Nacional de Terapia EMDR, que puede afiliarse a EMDR-Iberoamérica. Debido a limitaciones de espacio, no nos es posible describir en este artículo las iniciativas de entrenamiento que hemos desarrollado.

\section{Historias Clínicas del Trabajo de Campo con Terapia EMDR Después de Desastres}

\section{Historia Clínica \#1. En un pueblo de México después de un huracán}

Durante un ejercicio de grupo, la terapeuta pidió a los participantes que realizaran el abrazo de la mariposa y respiración profunda. Dos hermanos (de $18 \mathrm{y}$ 16 años de edad) que no realizaban el ejercicio, estaban situados a una corta distancia observando atentamente. Después de que terminó el ejercicio, el joven de 16 años se acercó a la terapeuta para contarle su historia y preguntarle cómo podría su hermano realizar el abrazo de la mariposa sin brazos.

Él le platicó que una noche, todos los miembros de su familia: padre, madre y los cuatro hijos (de 5, 7, 16 y 18 de edad), fueron alcanzados con gran furia por la corriente del río, que arrasó con todo lo que había a su paso. Primero, la corriente arrastró a sus padres y su casa. El joven de 18 años luchó con todas sus fuerzas para rescatar a sus tres hermanos, pero solo pudo rescatar al hermano de 16 años, porque el agua le arrebató a los otros dos de sus brazos. Este esfuerzo le dejó un gran daño físico en los brazos. Después de dos días, los dos jóvenes fueron rescatados y cuando finalmente llegaron al hospital, los brazos del joven de 18 años estaban gangrenados y tuvieron que ser amputados.

La terapeuta pidió al joven que la llevara con su hermano para mostrarles como podían hacer el abrazo de la mariposa. Ella le preguntó si creía que su hermano había realizado un enorme acto de amor al rescatarlo. El respondió inmediata y efusivamente que sí. Después, le pidió que se colocara detrás de su hermano mayor, quien estaba en una silla de ruedas, de tal manera que una de sus mejillas estuviera en contacto con la mejilla de su hermano, y que lo abrazara por detrás, cruzando sus brazos sobre el pecho del hermano. Posteriormente la terapeuta les pidió a ambos que respiraran profundamente y al joven de 16 años, que realizara el abrazo de la mariposa en el pecho de su hermano.

Los tres trabajaron con Terapia EMDR para reprocesar las memorias traumáticas mientras que realizaban el abrazo de la mariposa para la estimulación bilateral. No hay palabras para expresar la transformación del rostro de los hermanos, de desesperación a amor profundo. Este es uno de los actos de amor más grandes que la terapeuta haya presenciado.

\section{Historia Clínica \#2. En un pueblo de México después de un huracán}

Una joven de 17 años perdió su casa, a sus padres y a dos hermanos menores debido al huracán. Durante el escaneo corporal, ella reportó "Tengo un agujero en el pecho porque no puedo recordar las voces de mis padres y hermanos". La terapeuta pidió a la joven que realizara el abrazo de la mariposa, probando ritmos de diferente intensidad y velocidad. Después de un tiempo, la joven encontró cuatro diferentes ritmos, cada uno de ellos simbolizaba las voces de sus seres amados resonando en perfecta armonía en su cuerpo y en su corazón. Al final de la Terapia EMDR, la joven 
dijo: "Nunca más estaré sola, ahora puedo enfrentar mi futuro de una manera diferente y positiva. .."

Historia Clínica \#3. En un pueblo de Centroamérica devastado por un huracán

Una mujer de 39 años, contó al terapeuta que cuando la poderosa agua entró a su casa, su esposo les ayudó a ella y a sus dos hijos a alcanzar el techo, pero cuando él trató de alcanzarlos, el agua movió el refrigerador hacia donde estaba y quedó atrapado entre la pared y el refrigerador. Ella no permitió que sus hijos de $10 \mathrm{y}$ 7 años vieran a su padre; sin embargo, ella presenció en total impotencia, como el agua subió hasta ahogarlo, observando todo el tiempo, la mirada de terror de su esposo. Tres días después, fueron rescatados.

Su sufrimiento era terrible; además de sus reacciones de estrés postraumático, no había podido ver a sus hijos desde el día del rescate ( 3 semanas antes) porque ellos le activaban el aterrador recuerdo de la muerte de su esposo. Había dejado a los niños en casa de su hermana. El terapeuta y ella trabajaron con Terapia EMDR. Tres días después, la mujer regresó temprano en la mañana y le presentó al terapeuta a sus dos hijos.

Gracias al reprocesamiento de la memoria traumática, ella logró establecer nuevamente una relación con ellos sin que se activara ninguna memoria traumática. Ella solicitó al terapeuta que los niños también recibieran tratamiento y éstos fueron incluidos en un grupo de niños que trabajaron con el EMDR-IGTP. Al final del día, la mujer y sus hijos se despidieron y agradecieron al terapeuta, alejándose tomados de las manos.

\section{Historia Clínica \#4. En un pueblo del Caribe devastado por un huracán}

Un hombre de 59 años pidió ser atendido por un terapeuta hombre. En la entrevista, el dijo que la razón por la que había hecho esa petición era porque no quería llorar en frente de una mujer. Explicó que las aguas se llevaron su pobre y pequeño rancho matando a su esposa y a todos sus animales. Sus únicas posesiones materiales eran la ropa que llevaba puesta. Dijo que desde que sucedió el huracán no había llorado, para darles un ejemplo de fortaleza a sus hijos adultos y a sus nietos; pero que las memorias traumáticas y el dolor eran tan avasalladores que ya no tenía más fuerzas y estaba pensando en el suicidio.

El terapeuta trabajó con Terapia EMDR y al final de la sesión, el hombre comentó con vivacidad en su mirada: "Construiré mi casa nuevamente con mis propias manos y conseguiré una buena gallina ponedora; y con eso, me levantaré nuevamente y seré un ejemplo para mis hijos y mis nietos."
Historia Clínica \#5. En un pueblo de

Sudamérica después de un terremoto

Una niña de 10 años llamada María se estaba bañando cuando ocurrió el terremoto. El vidrio de la regadera se hizo añicos y le causó heridas en diferentes partes del cuerpo, especialmente en su pecho. Ella se vio sometida a diversas cirugías y tratamientos dolorosos. Además, había recibido insultos por parte de los niños y niñas de su escuela, tales como "eres un monstruo". Ella presentaba síntomas de trauma complejo cuando llegó por primera vez para solicitar apoyo de salud mental. La terapeuta trabajó con ella utilizando Terapia EMDR y el abrazo de la mariposa para la estimulación bilateral. En la última sesión, la niña abrió sus ojos y en una epifanía le dijo a la terapeuta, "Ahora sé porque me sucedió esto. . porque los niños que se hayan cortado, quemado o lastimado me van a creer cuando les diga que con la Terapia EMDR hay esperanza para ellos."

\section{Historia Clínica \# 6. En un pueblo de Sudamérica después de un huracán}

En un refugio, un niño de 12 años le contó a la terapeuta la peor imagen que tenía del desastre: "Yo tenía agarrada a mi hermana de 10 años de la mano, pero el agua me la arrebató y vi como ella se iba luchando contra el agua antes de desaparecer." El niño reprocesó esa terrible memoria con Terapia EMDR, pero ninguna terapia podía aliviar su dolor y la tristeza por la muerte de su hermana.

Dos días después, la terapeuta estaba en un hospital local aplicando Terapia EMDR a niños y niñas sobrevivientes del huracán. Durante la sesión de Terapia EMDR, una niña de 10 años le contó que el agua la había arrebatado de los brazos de su hermano de 12 años. Ella encontró la rama de un árbol y flotó sobre la rama río abajo durante dos días y una noche, llegando hasta el océano en donde fue rescatada. Durante el tratamiento, la niña reprocesó su peor recuerdo, el de casi ahogarse cuando su cara se sumergía cada vez que ella se quedaba dormida.

La terapeuta no podía creer que la similitud de las historias fuera una coincidencia, por lo que le pidió a la niña que le diera el nombre y apellido de su hermano y padres, sin decirle para que. Tan pronto como terminó, se fue al refugio, buscó al niño y le preguntó el nombre completo de sus padres. Eran los mismos. Le pidió al niño que la llevara con ellos y cuando estaban todos juntos, les dijo: "Su niña está sana y salva." No hay lenguaje que pueda describir las emociones que se vivieron en ese momento y cuando la familia se reunió en el hospital. 
Historia Clínica \#7. En una Ciudad del Caribe después de un terremoto devastador

Un hombre de 39 años con síntomas de estrés agudo solicitó tratamiento a un terapeuta EMDR que había llegado al sitio del desastre 12 días después del terremoto. El estaba hipervigilante y sentía pánico con cada nueva réplica. Experimentaba imágenes intrusivas, olores y sonidos del terremoto inicial y de los trabajos de rescate en los que había participado los días subsecuentes. Tenía pesadillas repetitivas del terremoto, en el que él y toda su familia morían, a pesar de que en realidad su familia había sobrevivido milagrosamente. El y su familia estaban viviendo en la calle, enfrente de su casa derrumbada y tenía temor de que les robaran o mataran. "Estoy perdiendo la razón. . . me estoy volviendo loco. . .no puedo tolerar más este infierno," le dijo al terapeuta con pánico en sus ojos.

El terapeuta utilizó Terapia EMDR para reprocesar las memorias traumáticas. Por razones de tiempo y de número de pacientes, únicamente le pudo dar una sesión de tratamiento. En un viaje posterior realizado dos meses después, el hombre pidió una cita con el terapeuta EMDR. La entrevista duró dos minutos, porque el hombre solo quería decirle, que durante los cinco días posteriores a su terapia EMDR, usó el abrazo de la mariposa cada vez que los síntomas intrusivos aparecían; y que después de esos cinco días, los síntomas habían desaparecido. "Fue un milagro. . . ya no tengo miedo de las réplicas y he podido conseguir un trabajo. . . quiero decirle que todos los días le agradezco a Jesús por haberlo traído aquí y le pido que lo proteja y que le dé una larga vida para aliviar el sufrimiento humano."

\section{Intervenciones Humanitarias con Terapia EMDR}

\section{Después de un Desastre Provocado por el Hombre}

Cuando el derrumbe de una mina mató a 65 mineros en el estado de Coahuila en México en 2006, el equipo de AMAMECRISIS viajó al lugar de los hechos para dar tratamiento a niños traumatizados y en duelo. Dieciséis niños huérfanos de padre participaron en el EMDR-IGTP. Los resultados mostraron una disminución significativa en los puntajes que obtuvieron en la Escala CRTES (Child's Reaction to Traumatic Events Scale) y estos resultados se mantuvieron en el seguimiento que se realizó 3 meses después (Jarero, Artigas $\&$ Montero, 2008; ver Figuras 1 y 2).

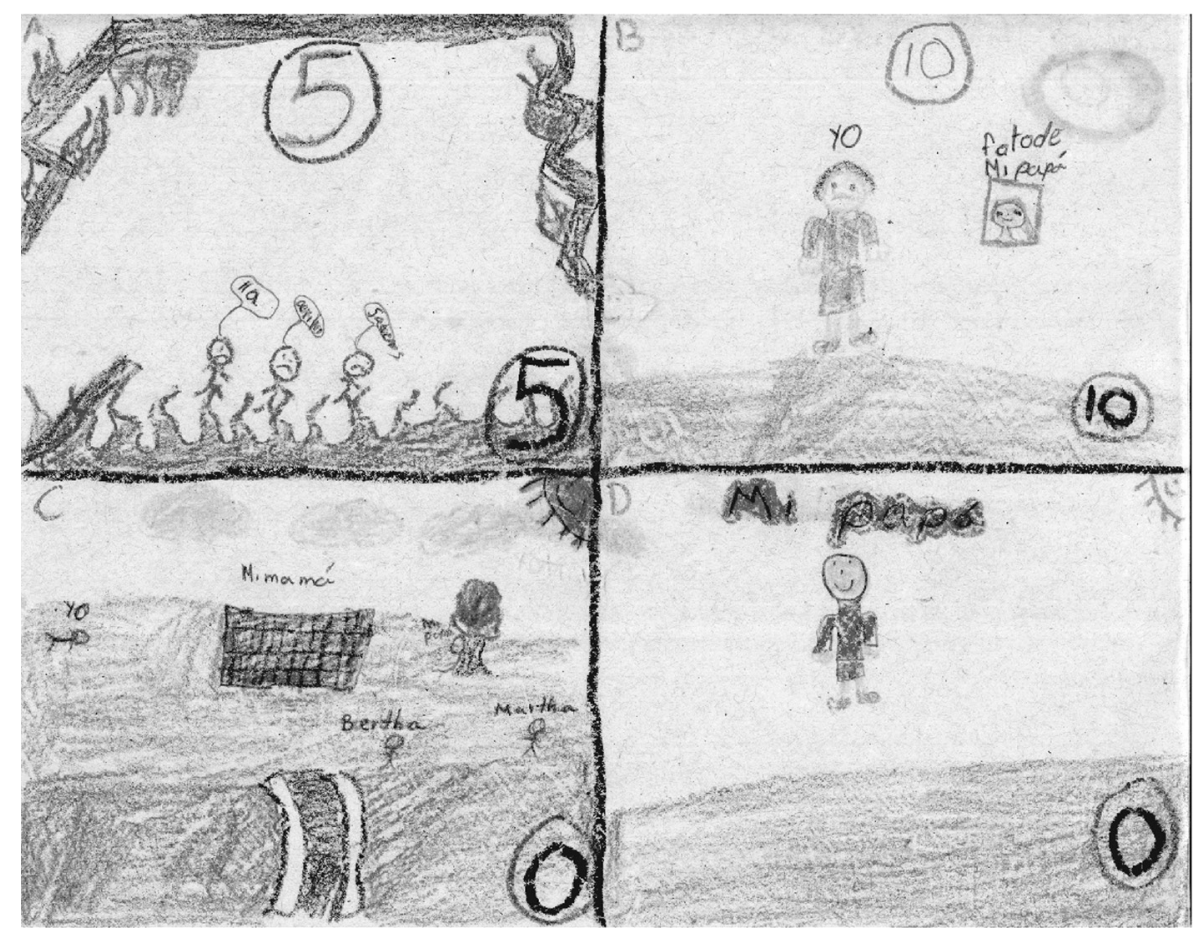

FIGURA 1. Ejemplo del dibujo de un niño antes y durante el tratamiento con el EMDR-IGTP. Los números representan los puntajes auto-reportados por el niño obtenidos en la Escala de Unidades Subjetivas de Perturbación (SUDS, por sus siglas en ingles). Dibujo A = Las figuras atrapadas adentro de la mina (su padre es una de ellas) están diciendo "Ha," "Auxilio," "Sálvenos" (SUDS = 5). Dibujo B = "Yo," "Foto de mi papá” (SUDS = 10). Dibujo C = "Yo", "Mi mamá," "Bertha," "Martha" (sus hermanas; SUDS = 0). Dibujo D = "Mi papá” (SUDS =0). Adaptado de Jarero, I., Artigas, L. \& Montero, M. (2008). The EMDR integrative group treatment protocol: Application with child victims of a mass disaster. Journal of EMDR Practice and Research, 2, 97 - 105. Autorizado para reimpresión. 


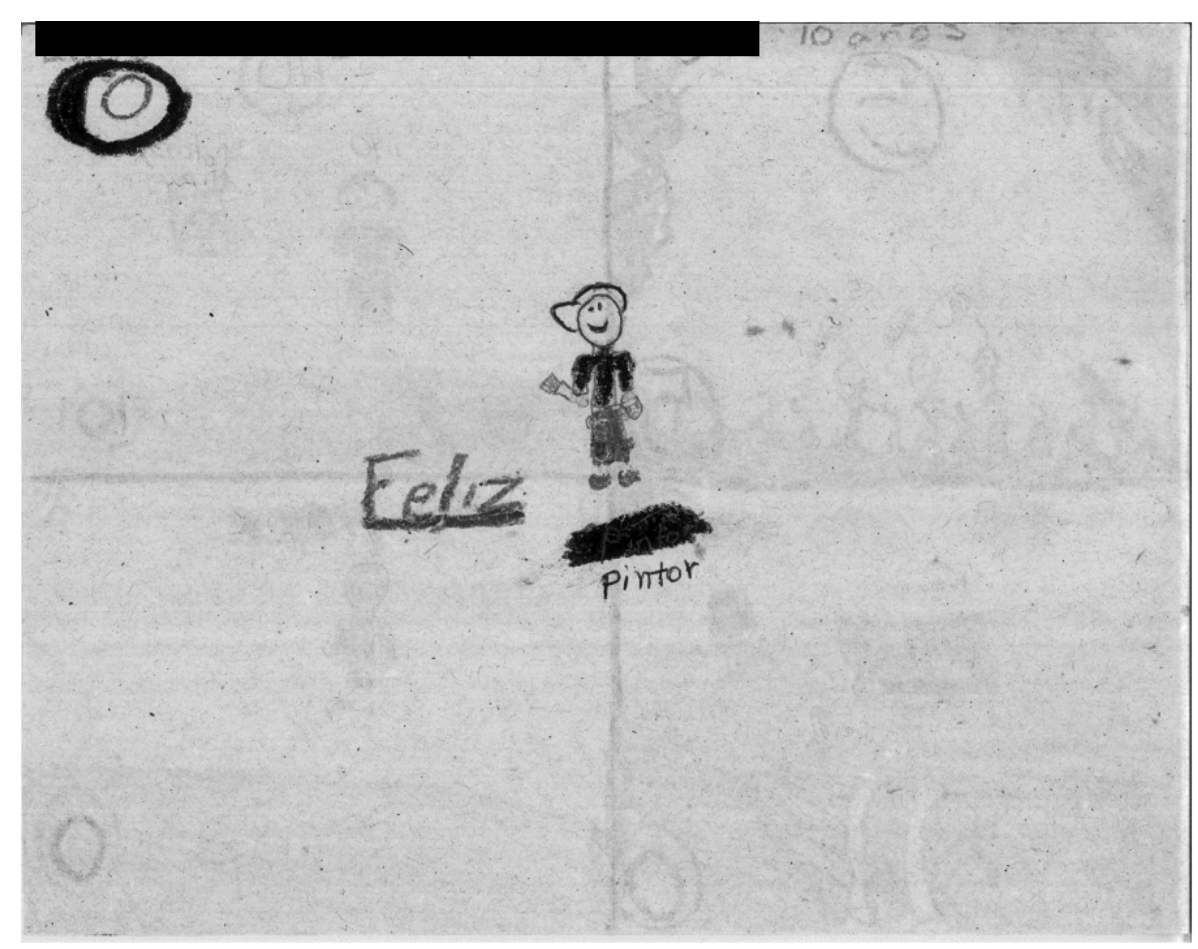

FIGURA 2. Ejemplo del futuro imaginado por el niño. "Feliz", "Pintor." (SUDS=0). Adaptado de Jarero, I., Artigas, L. \& Montero, M. (2008). The EMDR integrative group treatment protocol: Application with child victims of a mass disaster. Journal of EMDR Practice and Research, 2, 97 - 105. Autorizado para reimpresión.

\section{Después de un Terremoto de 7.2 grados de Magnitud}

Esta intervención se llevó a cabo después de un terremoto de 7.2 grados de magnitud, en el estado de Baja California Norte en México. El tratamiento se aplicó de acuerdo a los principios del continuo de cuidados en salud mental. Una sesión de tratamiento con el EMDR-PRECI (Jarero et al., 2011; Jarero \& Uribe, $2011,2012)$ produjo una mejoría significativa en los síntomas de estrés postraumático en los dos grupos atendidos: grupo de atención inmediata y grupo de atención demorada. Estos resultados se mantuvieron en la evaluación de seguimiento que se realizó 12 semanas después, a pesar de que continuaron presentándose fuertes réplicas de manera frecuente.

\section{Después de una Masacre Humana}

Después de una masacre humana en el estado de Durango en México, el personal forense tuvo la terrible tarea de recuperar 258 cuerpos mutilados de fosas clandestinas. Durante este proceso que duró meses, ellos estuvieron expuestos continuamente a terribles estresores emocionales, que incluyeron amenazas del crimen organizado contra su propia integridad y la de sus familias. Se administró una sesión con Terapia
EMDR a 32 trabajadores forenses. Los resultados mostraron una mejoría significativa en los dos grupos a los que se les aplicó el tratamiento: grupo de atención inmediata y grupo de atención demorada (Jarero \& Uribe, 2011, 2012) en la Escala de Impacto del Evento (EIE) y en la Escala Short PTSD Rating Interview (SPRINT; Connor \& Davidson, 2001; Vaishnavi, Payne, Connor \& Davidson, 2006).

\section{Para Víctimas de Violencia Interpersonal Severa}

Durante 2011, 2012 y 2013, AMAMECRISIS trabajó con la Organización Inocencia en Peligro - Colombia, llevando a cabo en ese país varios campamentos de recuperación del trauma de 3 semanas de duración cada uno, para un total de 89 niños y niñas, de entre los 9 y 17 años de edad, quienes habían sido víctimas de trauma interpersonal severo (ej. violación, abuso sexual, violencia física y psicológica, negligencia y abandono). En estos campamentos se administró una aproximación terapéutica multi-componente y en fases para el tratamiento del trauma, la cual incluyó Terapia EMDR individual y una versión modificada del EMDR-IGTP adaptada para trauma interpersonal severo.

Los resultados obtenidos en el SPRINT para todos los grupos, mostraron una mejoría estadísticamente 
significativa después del tratamiento, la cual continuó incrementándose hasta la medida de seguimiento (Jarero, Roque-López \& Gómez, 2013; Jarero, Roque-López, Gómez \& Givaudán, 2014a, 2014b). La reducción de los síntomas de TEPT se atribuyeron principalmente a la Terapia EMDR, ya que las actividades que se realizaron antes de las sesiones de reprocesamiento con Terapia EMDR (ej. deporte, gimnasia suave, hatha yoga, contar cuentos, pintura, danza, teatro y mindfullness) no mostraron haber causado una reducción estadísticamente significativa en la severidad de los síntomas de TEPT medidos con el SPRINT (Jarero et al., 2014b)

\section{Para Pacientes con Cáncer}

En el 2013, el primer autor (IJ) recibió el diagnóstico de cáncer de próstata y desarrolló síntomas de estrés postraumático relacionados con el cáncer, los cuáles fueron tratados exitosamente con Terapia EMDR. Motivado por su experiencia personal y como co-fundador de AMAMECRISIS, en 2014 ofreció a la Organización Mexicana "Cruz Rosa," realizar un estudio piloto y dar Terapia EMDR grupal a 24 pacientes mujeres con diferentes tipos de cáncer (ej. cervicouterino, de mama, colon, hígado y piel) en Monterrey, México. Todas la mujeres, en edades entre los $38 \mathrm{y}$ 68 años $(\mathrm{M}=54.2)$, habían recibido el diagnóstico de cáncer entre julio del año 2006 y octubre del 2013, reportaban síntomas de estrés postraumático relacionado al cáncer y estaban en diferentes etapas del tratamiento.

Los terapeutas EMDR administraron 6 sesiones del EMDR-IGTP adaptado para estrés traumático continuado, durante 3 días consecutivos (una sesión en la mañana y una en la tarde). Los resultados mostraron una reducción estadísticamente significativa en los puntajes del SPRINT obtenidos por las participantes entre el pre-tratamiento y el primer seguimiento y el pre-tratamiento y el segundo seguimiento, realizado 3 meses después (Jarero, Artigas, Uribe, García, Cavazos \& Givaudán, 2014). Actualmente, AMAMECRISIS y la Fundación Latinoamericana y del Caribe para la Investigación del Trauma Psicológico están diseñando proyectos de investigación con estudios aleatorios y controlados para esta población y sus familias.

\section{Para Auxiliadores}

En el año 2012, AMAMECRISIS trató a 39 auxiliadores en servicio activo que fueron asignados al azar para recibir dos sesiones de 90 minutos de tratamiento con el EMDR-PROPARA (Jarero et al., 2013) o con Consejería de Apoyo.

Los participantes del grupo tratado con el EMDRPROPARA mostraron beneficios inmediatamente después del tratamiento, observándose además, una mayor disminución en los puntajes del SPRINT en el segundo seguimiento realizado tres meses después. En comparación, en el grupo de Consejería de Apoyo, los participantes experimentaron una disminución no significativa después del tratamiento y un aumento en los puntajes del SPRINT en el segundo seguimiento.

\section{Discusión}

Una consecuencia de los desastres, que frecuentemente se subestima, son las heridas psicológicas, las cuales no siempre son visibles, agudas o atendidas. Durante más de 10 años, los expertos han concluido que los daños psicológicos de un desastre pueden sobrepasar a los físicos en una proporción de 4 a 1 (Everly, Barnett, Sperry \& Links, 2010). Los protocolos para Intervención Temprana con Terapia EMDR, son intervenciones breves con efectos de tratamiento rápidos, que pueden ser utilizados en campo o en situaciones de emergencia y que pueden reducir el número de víctimas de daños psicológicos.

\section{Pasado, Presente y Futuro}

Hace 16 años, cuando vimos en televisión la destrucción y el sufrimiento que había causado el Huracán Paulina a nuestros hermanos y hermanas mexicanas, sentimos la enorme necesidad de estar con ellos y ofrecer nuestra ayuda. Nunca imaginamos que dejar la comodidad de nuestras oficinas en la Ciudad de México para ir a trabajar con niños bajo un árbol de mango y altas temperaturas, nos pudiera haber dado la experiencia más gozosa de nuestra vida profesional. En el viaje de regreso a casa, tomamos la decisión de dedicar nuestras vidas a aliviar el sufrimiento humano provocado por el trauma psicológico.

En este sentido hemos continuado, ya sea trabajando directamente en campo o apoyando a colegas de otros países alrededor del mundo para ayudar a aquellos que han sobrevivido algún desastre. Invitamos a todos nuestros colegas terapeutas EMDR alrededor del mundo, a no esperar a que ocurra un desastre masivo para abandonar sus consultorios. En estos días, en su propia ciudad, existe lo que llamamos "desastres urbanos" (ej. pacientes con cáncer, inmigrantes, violencia interpersonal severa). Ustedes pueden utilizar los protocolos de Terapia EMDR que han surgido 
como resultado de nuestro trabajo de campo y que han sido validados como efectivos (ej. EMDR-IGTP, EMDR-PRECI). Solo necesitan ir con los sobrevivientes que están sufriendo - ellos los están esperando.

Queremos compartir con todos ustedes estas alentadoras palabras que la Dra. Francine Shapiro escribió al equipo de AMAMECRISIS el día que recibimos el Premio Francine Shapiro en la ciudad de Brasilia, en el año 2007:

... Y si otros siguieran sus pasos y realizaran la investigación aleatoria y controlada que se necesita para consolidar el trabajo ante los ojos del mundo, y poderlo declarar "empíricamente validado" por las organizaciones internacionales de gran envergadura como la UNICEF, entonces miles y miles más serían sanados en los años venideros.

\section{Referencias}

Adúriz, M. E., Knopfler, C., \& Bluthgen, C. (2009). Helping child flood victims using group EMDR intervention in Argentina: Treatment outcome and gender differences. International Journal of Stress Management, 16 (2), 138-153.

Adúriz, M. E., \& Salas, C. (2014). Aplicación del Protocolo Grupal e Integrativo con EMDR a las Víctimas de un Alud en Tartagal-Salta-Argentina. Revista Iberoamericana de Psicotraumatología y Disociación, 6 (2), 1-12. ISSN: 2007-8544.

Artigas, L., \& Jarero, I. (2014). The butterfly hug. In M. Luber (Ed.), Implementing EMDR early mental health interventions for man-made and natural disasters (pp. 127-130). New York, NY: Springer Publishing.

Artigas, L., Jarero, I., Alcalá, N., \& López Cano, T. (2014). The EMDR Integrative Group Treatment Protocol (IGTP) for children. In M. Luber (Ed.), Implementing EMDR early interventions for man-made and natural disasters (pp. 237-251). New York, NY: Springer Publishing.

Artigas, L., Jarero, I., Mauer, M., López Cano, T., \& Alcalá, N. (2000, September). EMDR and traumatic stress after natural disasters: Integrative treatment protocol and the butterfly hug. Poster presented at the EMDRIA Conference, Toronto, Ontario, Canada.

Biles, J., \& Cobos D. (2004). Chapter 15: Natural disasters and their impact in Latin America. In J. P. Stoltman, J. Lidstone, \& L. M. DeChano (Eds.), Advances in Natural and Technological Hazards Research Series: Vol. 21. International perspectives on natural disasters: Occurrence, mitigation, and consequences (pp. 281-302). New York, NY.

Bisson, J., \& Andrew, M. (2007). Psychological treatment of post-traumatic stress disorder (PTSD). Cochrane Database of Systematic Reviews, (3), CD003388.

Connor, K. M., \& Davidson, J. R. T. (2001). SPRINT: A brief global assessment of post-traumatic stress disorder. International Clinical Psychopharmacology, 16 (5), 279-284.

Everly, G. S., Jr., Barnett, D. J., Sperry, N. L., \& Links, J. M. (2010). The use of psychological first aid (PFA) training among nurses to enhance population resiliency. International Journal of Emergency Mental Health, 12 (1), 21-31.

Fernandez, I., Gallinari, E., \& Lorenzetti, A. (2005). A school-based intervention for children who witnessed the Pirelli building airplane crash in Milan, Italy. Journal of Brief Therapy, 2, 129-136.

Gelbach, R., \& Davis, K. (2007). Disaster response: EMDR and family systems therapy under community wide stress. In F. Shapiro, F. W. Kaslow, \& L. Maxfield (Eds.), Handbook of EMDR and family therapy processes (pp. 387-406). New York, NY: Wiley.

Green Cross Academy of Traumatology. (2008). Standards of self-care guidelines. Retrieved from http: / / compassion fatigue.dreamhosters.com/wp-content/uploads / 2010/11/academy-ethical.pdf

Jarero, I., Amaya, C., Givaudán, M., \& Miranda, A. (2013). EMDR individual protocol for paraprofessional use: A randomized controlled trial with first responders. Journal of EMDR Practice and Research, 7 (2), 55-64.

Jarero, I., \& Artigas, L. (2009). EMDR integrative group treatment protocol. Journal of EMDR Practice \& Research, 3 (4), 287-288.

Jarero, I., \& Artigas, L. (2010). EMDR integrative group treatment protocol: Application with adults during ongoing geopolitical crisis. Journal of EMDR Practice and Research, 4 (4), 148-155.

Jarero, I., \& Artigas, L. (2012). The EMDR integrative group treatment protocol: EMDR group treatment for early intervention following critical incidents. European Review of Applied Psychology, 62, 219-222.

Jarero, I., Artigas, L., \& Hartung, J. (2006). EMDR integrative treatment protocol: A post-disaster trauma intervention for children \& adults. Traumatology, 12, 121-129.

Jarero, I., Artigas, L., \& Luber, M. (2011). The EMDR protocol for recent critical incidents: Application in a disaster mental health continuum of care context. Journal of EMDR Practice and Research, 5 (3), 82-94.

Jarero, I., Artigas, L., Mauer, M., López Cano, T., \& Alcalá, N. (1999, November). Children's post-traumatic stress after natural disasters: Integrative treatment protocol. Poster session presented at the annual meeting of the International Society for Traumatic Stress Studies, Miami, FL.

Jarero, I., Artigas, L., \& Montero, M. (2008). The EMDR integrative group treatment protocol: Application with child victims of a mass disaster. Journal of EMDR Practice and Research, 2, 97-105.

Jarero, I., Artigas, L., Uribe, S., García, L, E., Cavazos, M. A., \& Givaudán, M. (2014). Estudio Piloto sobre la Aplicación del Protocolo Grupal e Integrativo con Terapia EMDR en Mujeres con Cáncer. Revista Iberoamericana de Psicotraumatología y Disociación, 6(3), 1-17, ISSN: 2007-8544.

Jarero, I., Roque-López, S., \& Gómez, J. (2013). The provision of an EMDR-based multicomponent trauma treatment with child victims of severe interpersonal trauma. Journal of EMDR Practice \& Research, 7 (1), 17-28.

Jarero, I., Roque-López, S., Gómez, J., \& Givaudán, M. (2014a). Second research study on the provision of 
the EMDR integrative group treatment protocol with child victims of severe interpersonal violence. Revista Iberoamericana de Psicotraumatología y Disociación, 6 (1), 1-24. ISSN: 2007-8544.

Jarero, I., Roque-López, S., Gómez, J., \& Givaudán, M. (2014b). Third research study on the provision of the EMDR integrative group treatment protocol with child victims of severe interpersonal violence. Revista Iberoamericana de Psicotraumatología y Disociación, 6 (2), 1-22. ISSN: 2007-8544.

Jarero, I., \& Uribe, S. (2011). The EMDR protocol for recent critical incidents: Brief report of an application in a human massacre situation. Journal of EMDR Practice and Research, 5 (4), 156-165.

Jarero, I., \& Uribe, S. (2012). The EMDR protocol for recent critical incidents: Follow-up report of an application in a human massacre situation. Journal of EMDR Practice and Research, 6 (2), 50-61.

Jarero, I., \& Uribe, S. (2014). Worst case scenarios in recent trauma response. In M. Luber (Ed.), Implementing EMDR early mental health interventions for man-made and natural disasters (pp. 533-541). New York, NY: Springer Publishing.

Luber, M. (2009). EMDR and early interventions for groups. In M. Luber (Ed.), Eye movement desensitization and reprocessing (EMDR) scripted protocols: Basic and special situations (pp. 277-278). New York, NY: Springer Publishing.

Maxfield, L. (2008). EMDR treatment of recent events and community disasters. Journal of EMDR Practice \& Research, 2 (2), 74-78.

Mojica, S., Márquez, O., Guadarrama, R., \& Ramos, L. (2013). Measurement of posttraumatic stress disorder (PTSD) in Mexican university students. Salud Mental, 36, 451-461. Retrieved from http://saludmental.edilaser.net/en/ pdf/3606/SM_13.06.07_EN_p451-461.pdf

Monteiro, A. (2014). Group and individual EMDR therapy in the humanitarian assistance project in Southern Brazil. Revista Iberoamericana de Psicotraumatología y Disociación, 6 (3), 1-19. ISSN: 2007-8544.
Norris, F. H., Murphy, A. D., Baker, C. K., \& Perilla, J. L. (2004). Postdisaster PTSD over four waves of a panel study of Mexico's 1999 flood. Journal of Traumatic Stress, 17 (4), 283-292.

Oren, E., \& Solomon, R. (2012). EMDR therapy: An overview of its development and mechanisms of action. European Review of Applied Psychology, 62, 197-203.

Pan American Health Organization. (2012). Health in the Americas 2012. Chapter 3: The environment and human security. Retrieved from http://www.paho.org/saluden lasamericas $/$ index.php?option $=$ com_content $\&$ view $=$ article $\&$ id $=568$ Itemid $=52 \&$ lang $=$ en

Salas, C. (2014). Aplicación del Protocolo Grupal e Integrativo con EMDR a las Víctimas de un Alud en AngastacoArgentina. Revista Iberoamericana de Psicotraumatología y Disociación, 6 (2), 1-11. ISSN: 2007-8544.

Shapiro, F. (2001). Eye movements, desensitization and reprocessing: Basic principles, protocols, and procedures (2nd Ed.). New York, NY: Guilford Press.

Solomon, R., \& Shapiro, F. (2008). EMDR and the adaptive information processing model: Potential mechanism of change. Journal of EMDR Practice and Research, 2 (4), 315-325.

Vaishnavi, S., Payne, V., Connor, K., \& Davidson, J. R. (2006). A comparison of the SPRINT and CAPS assessment scales for posttraumatic stress disorder. Depression and Anxiety, 23 (7), 437-440.

World Health Organization. (2013). Guidelines for the managements of conditions specifically related to stress. Geneva, Switzerland: Author.

Zaghrout-Hodali, M., Alissa, F., \& Dodgson, P. (2008). Building resilience and dismantling fear: EMDR group protocol with children in an area of ongoing trauma. Journal of EMDR Practice and Research, 2 (2), 106-113.

La Correspondencia referente a este artículo, debe de ser dirigida a Ignacio Jarero, PhD, Ed.D. Boulevard de la Luz 771, Jardines del Pedregal, Álvaro Obregón, México City 01900. E-mail: nacho@amamecrisis.com.mx 